\title{
Recent highlights from the ATLAS heavy-ion program
}

\author{
Aaron Angerami on behalf of the ATLAS Collaboration ${ }^{1}$ \\ Columbia University, New York, NY 10027, USA
}

\begin{abstract}
These proceedings report on recent measurements performed with the ATLAS Detector at the LHC presented at the Quark Matter 2015 Conference. These include measurements of two-particle correlations in $p p$ collisions at $\sqrt{s}=2.76$ and $13 \mathrm{TeV}$. These results are the first to show a second harmonic modulation in the azimuthal dependence of the particle yields in $p p$ collisions, a signature closely associated with collective flow in ion-ion collisions. Measurements of $p+\mathrm{Pb}$ collisions at $\sqrt{s_{\mathrm{NN}}}=5.02 \mathrm{TeV}$ are presented including charged particle multiplicities, Bose-Einstein correlations and yields of jets and electroweak bosons that provide insight into the initial stages of these collisions including the nuclear geometry. Finally, measurements of dijet asymmetry and jet structure in $\mathrm{Pb}+\mathrm{Pb}$ and $p p$ collisions at $\sqrt{s_{\mathrm{NN}}}=2.76 \mathrm{TeV}$ are presented that further illuminate the phenomenon of partonic energy loss.
\end{abstract}

Keywords: ATLAS, heavy-ion collisions, quark-gluon plasma, jet quenching, hydrodynamics, elliptic flow

\section{Introduction}

Relativistic heavy-ion collisions provide a unique opportunity to study the most intriguing aspects of the strong interaction in the laboratory. In high energy ion-ion collisions studied at RHIC and the LHC, the energy densities are sufficiently high to produce a new form of matter referred to as the quark-gluon plasma [1-4]. This matter has been observed to quickly thermalize and evolve as a fluid with small viscosity. Since these fluid-like properties were discovered, much theoretical progress has been made in using viscous relativistic hydrodynamics to describe flow observables with ever-improving accuracy. Recently, signatures typically associated with collectivity in ion-ion collisions have also been observed in $p+\mathrm{Pb}$ collisions at the LHC and $d+\mathrm{Au}$ collisions at RHIC [5-15]. This unexpected result has raised questions about the applicability of hydrodynamics in small systems and underscores the fact that the mechanism by which the medium achieves local thermal equilibrium is not well understood. This has resulted in an increased interest in observables sensitivity to the initial stages of these collisions that can shed light on this question.

In addition to its collective expansion, the quark-gluon plasma has also been observed to be opaque to the passage of coloured particles, indicating that the medium is composed of a high density of unscreened color charges. The phenomenon of jet quenching - where jets produced in the earliest stages of the collision lose energy or have their parton showers modified by the presence of the medium - was another key discovery

\footnotetext{
${ }^{1} \mathrm{~A}$ list of members of the ATLAS Collaboration and acknowledgements can be found at the end of this issue.
} 
of the RHIC program [16-21]. The first heavy-ion jet measurements at the LHC revealed a large fraction of highly asymmetric dijet pairs in central $\mathrm{Pb}+\mathrm{Pb}$ collisions, providing the first direct observation of jet quenching and sparking an exciting era in the subject of partonic energy loss [22, 23].

These proceedings present a summary of recent measurements performed by the ATLAS Collaboration at the LHC [24] that bear directly on these questions and are organized as follows. Section 2 presents new measurements of two-particle correlations in $p p$ collisions. For the first time, an elliptic modulation to the particle yield as a function of azimuthal angle is observed in these collisions [25]. In Section 3, measurements in $p+\mathrm{Pb}$ collisions are presented that concern the relationship between the initial collision geometry and particle production. In Section 4, new ATLAS measurements in $\mathrm{Pb}+\mathrm{Pb}$ collisions sensitive to jet quenching are presented. These results are summarised in Section 5.

\section{Collective dynamics in small systems}

The observation of harmonic flow was a key factor supporting the interpretation that the nuclear matter formed in ion-ion collisions evolves hydrodynamically [1-4]. The flow results from spacial anisotropies in the initial density distribution of nuclear matter that are transformed into azimuthal anisotropies in the momentum spectra of the final particles. As most nuclear collisions possess an ellipsoidal overlap region, this phenomenon is most directly observed in the second harmonic modulation to the momentum spectra, so-called elliptic flow and is typically quantified by the magnitude of the second Fourier coefficient, $v_{2}$. Higher harmonic modulations have also been observed and are attributed to event-by-event fluctuations in the initial densities [26].

More recently harmonic modulation has been observed in $p+\mathrm{Pb}$ collisions. Although extracting a signal is more complicated in such systems as other azimuthal correlations, such as those arising from jet-like structures, have a larger contribution relative to the soft bulk carrying the flow information. To remove the effects of such correlations, experiments have utilized a recoil subtraction method applied to two-particle correlations (2PC), in which the shape of the dijet contribution to the $2 \mathrm{PC}$ is estimated in events with small activity (peripheral collisions) [5]. This background is then subtracted from the 2PCs obtained in more central collisions with the normalization of the background fixed by the Zero Yield at Minimum (ZYAM) requirement: after subtraction the yield should be zero at the minimum value of the correlation function $[21,27]$. A fit is then performed to the subtracted correlations to obtain the amplitudes of the various Fourier harmonics.

Recently, ATLAS has proposed a new method to extract the modulation by constructing a template using the peripheral reference and performing a fit to the correlations [25]. This method does not utilise the ZYAM procedure, which may introduce a bias if harmonic modulation is present in the peripheral reference itself. ATLAS has applied this template method to 2PCs measured in $p p$ collisions at $\sqrt{s}=2.76$ and $13 \mathrm{TeV}$. The correlation functions, defined as $C(\Delta \eta, \Delta \phi)=S(\Delta \eta, \Delta \phi) / B(\Delta \eta, \Delta \phi)$, were measured differentially in the transverse momentum ${ }^{2}$ of each particle, $p_{\mathrm{T}}^{a}$ and $p_{\mathrm{T}}^{b}$. Here $S$ is the pair yield and $B$ is an estimate of the combinatoric background obtained using an event mixing procedure. One-dimensional correlation functions, $C(\Delta \phi)$, were obtained by integrating the numerator and denominator over the long-range part of the correlation function, $2<|\Delta \eta|<5$. The large $\eta$ separation suppresses the contribution at small $\Delta \phi$ from jets. The $C(\Delta \phi)$ are converted into per-trigger-particle yields, $Y(\Delta \phi)$, according to :

$$
Y(\Delta \phi)=\left(\frac{\int \mathrm{d} \Delta \phi B(\Delta \phi)}{N^{a} \int \Delta \phi}\right) C(\Delta \phi),
$$

where $N^{a}$ denotes the efficiency-corrected number of trigger particles [5, 6, 21]. The $Y(\Delta \phi)$ are then fit using the template:

$$
Y^{\text {templ }}(\Delta \phi)=F Y^{\text {periph }}(\Delta \phi)+G\left(1+2 v_{2,2} \cos 2 \Delta \phi\right)
$$

\footnotetext{
${ }^{2}$ ATLAS uses a right-handed coordinate system with its origin at the nominal interaction point (IP) in the center of the detector and the $z$-axis along the beam pipe. The $x$-axis points from the IP to the center of the LHC ring, and the $y$-axis points upward. Cylindrical coordinates $(r, \phi)$ are used in the transverse plane, $\phi$ being the azimuthal angle around the beam pipe. The pseudorapidity is defined in terms of the polar angle $\theta$ as $\eta=-\ln \tan (\theta / 2)$.
} 

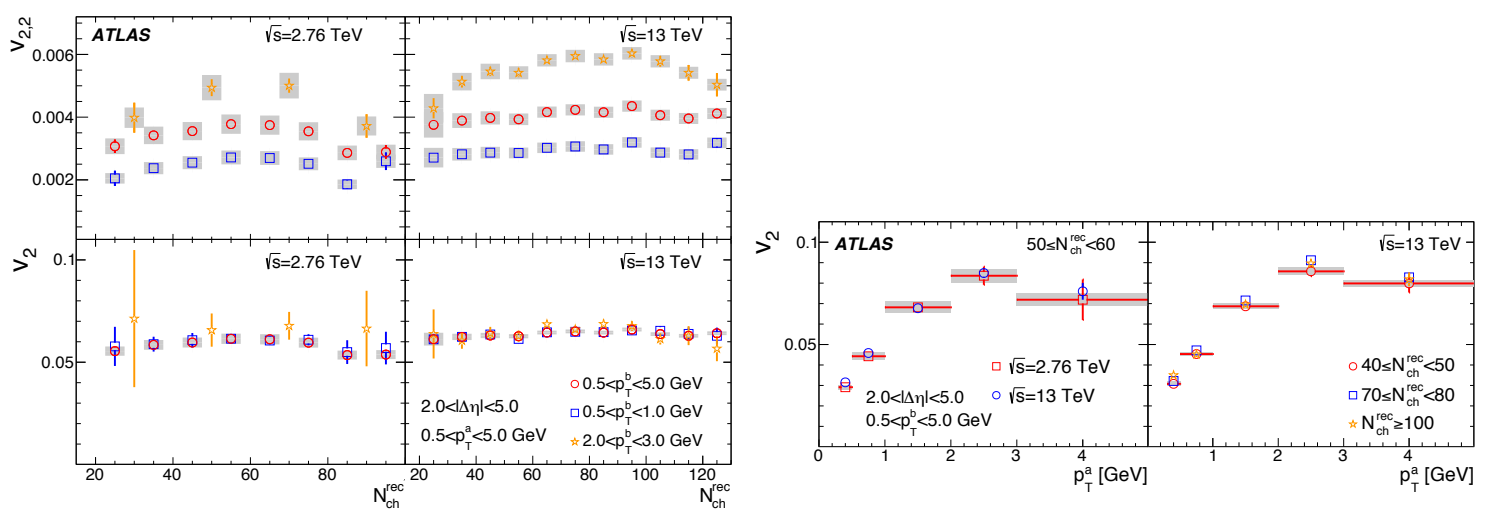

Fig. 1. Left: Measured $v_{2,2}$ (top) and $v_{2}$ (middle) values versus $N_{\mathrm{ch}}^{\text {rec }}$ for different $p_{\mathrm{T}}^{a}-p_{\mathrm{T}}^{b}$ intervals for 2.76 (left) and $13 \mathrm{TeV}$ (right) data. Results are averaged over $N_{\mathrm{ch}}^{\text {rec }}$ bins of width 10 spanning the range $20<N_{\mathrm{ch}}^{\text {rec }}<100$ and $20<N_{\mathrm{ch}}^{\text {rec }}<130$ for 2.76 and $13 \mathrm{TeV}$ data, respectively, except for the $2.0<p_{\mathrm{T}}^{b}<3.0 \mathrm{GeV}$ results for the $2.76 \mathrm{TeV}$ data which are averaged over bins of width 20 . Right: Measured $v_{2}$ values versus $p_{\mathrm{T}}^{a}$ for 13 and $2.76 \mathrm{TeV}$ data for the $50 \leq N_{\mathrm{ch}}^{\mathrm{rec}}<60$ interval (left) and for three $N_{\mathrm{ch}}^{\text {rec }}$ intervals in the $13 \mathrm{TeV}$ data (right). Results are averaged over the $p_{\mathrm{T}}^{a}$ intervals indicated by horizontal error bars. On all points, the vertical error bars indicate statistical uncertainties. The shaded bands indicate systematic uncertainties. For clarity, they are only shown for the $0.5<p_{\mathrm{T}}^{b}<5.0$ $\mathrm{GeV}$ case in the middle, for $2.76 \mathrm{TeV}$ data in the lower left, and for the $40 \leq N_{\mathrm{ch}}^{\text {rec }}<50$ case in the lower right panels. Taken from Ref. [25].

where the parameters $F$ and $v_{2,2}$ are determined by the fit and $G$ is fixed by the overall normalization. If the modulation arises from the single particle distributions alone, then $v_{2,2}\left(p_{\mathrm{T}}^{a}, p_{\mathrm{T}}^{b}\right)=v_{2}\left(p_{\mathrm{T}}^{a}\right) v_{2}\left(p_{\mathrm{T}}^{b}\right)$ where $v_{2}$ is the second Fourier coefficient of the single-particle azimuthal distribution. If this factorisation holds then the quantity, $v_{2}\left(p_{\mathrm{T}_{1}}\right)=v_{2,2}\left(p_{\mathrm{T}_{1}}, p_{\mathrm{T}_{2}}\right) / \sqrt{v_{2,2}\left(p_{\mathrm{T}_{1}}, p_{\mathrm{T}_{2}}\right)}$ should be independent of $p_{\mathrm{T}_{2}}$. The $v_{2,2}$ and $v_{2}$ values obtained in this fashion for various $p_{\mathrm{T}}$ selections are shown as a function of $N_{\mathrm{ch}}^{\mathrm{rec}}$ in the upper left panels of Figure 1 for the two collision energies. The $v_{2}$ values exhibit the factorisation property, indicating the modulation is present at the level of the single-particle distributions. The right two panels of Figure 1 show the $p_{\mathrm{T}}$ dependence of the $v_{2}$ values. The left panel compares the $p_{\mathrm{T}}$ dependence for $50<N_{\mathrm{ch}}^{\text {rec }}<60$ for the two different $\sqrt{s}$ values while the right compares the different $N_{\text {ch }}^{\text {rec }}$ ranges for $\sqrt{s}=13 \mathrm{TeV}$. The $p_{\mathrm{T}}$ dependence, specifically the linear rise and eventual turn over at $p_{\mathrm{T}} \sim 3 \mathrm{GeV}$ is characteristic of the trend observed in ion-ion collisions and closely associated with the hydrodynamic interpretation.

One possible explanation for this behavior is that rare collisions with the most activity are capable of producing matter similar to that produced in $\mathrm{Pb}+\mathrm{Pb}$ collisions, e.g. a miniature quark-gluon plasma [28]. In the ATLAS measurement, the $p_{\mathrm{T}}$ dependence is observed to be nearly independent of both $N_{\mathrm{ch}}^{\mathrm{rec}}$ and $\sqrt{s}$, indicating that the phenomenon is not significantly dependent on either total event activity or collision energy. This fact clearly shows that events with this type of correlation are fairly common in $p p$ collisions at LHC energies; the $v_{2}$ is non-zero over the entire $N_{\mathrm{ch}}^{\text {rec }}$ range, suggesting that either the dynamics governing the evolution of the quark-gluon-plasma are also relevant in typical $p p$ collisions or that the correlation has a different, possibly non-hydrodynamic, origin.

It should be noted that this observation of elliptic modulation in $p p$ collisions does not invalidate the interpretation of a similar observation in ion-ion collisions as flow; in that system a mechanism is still required to align the phases of the modulation of individual nucleon-nucleon collisions to produce the observed global correlation. Conversely, these results alone not prove the formation of a fluid-like medium. Detailed measurements of observables sensitive to hydrodynamics must be performed for this conclusion can be supported. A key question faced in applying hydrodynamics to such small systems is whether the scales in these systems are such that equations derived in the hydrodynamic limit remain valid or suffer from large higher order corrections. More practically, to form a predictive model such equations require initial conditions including descriptions of the event-by-event fluctuations in the initial density of the matter. The role of the geometry in these systems and its relationship to particle production is not fully understood. However, additional measurements of $p+\mathrm{Pb}$ collisions such as those presented in the following sections may 

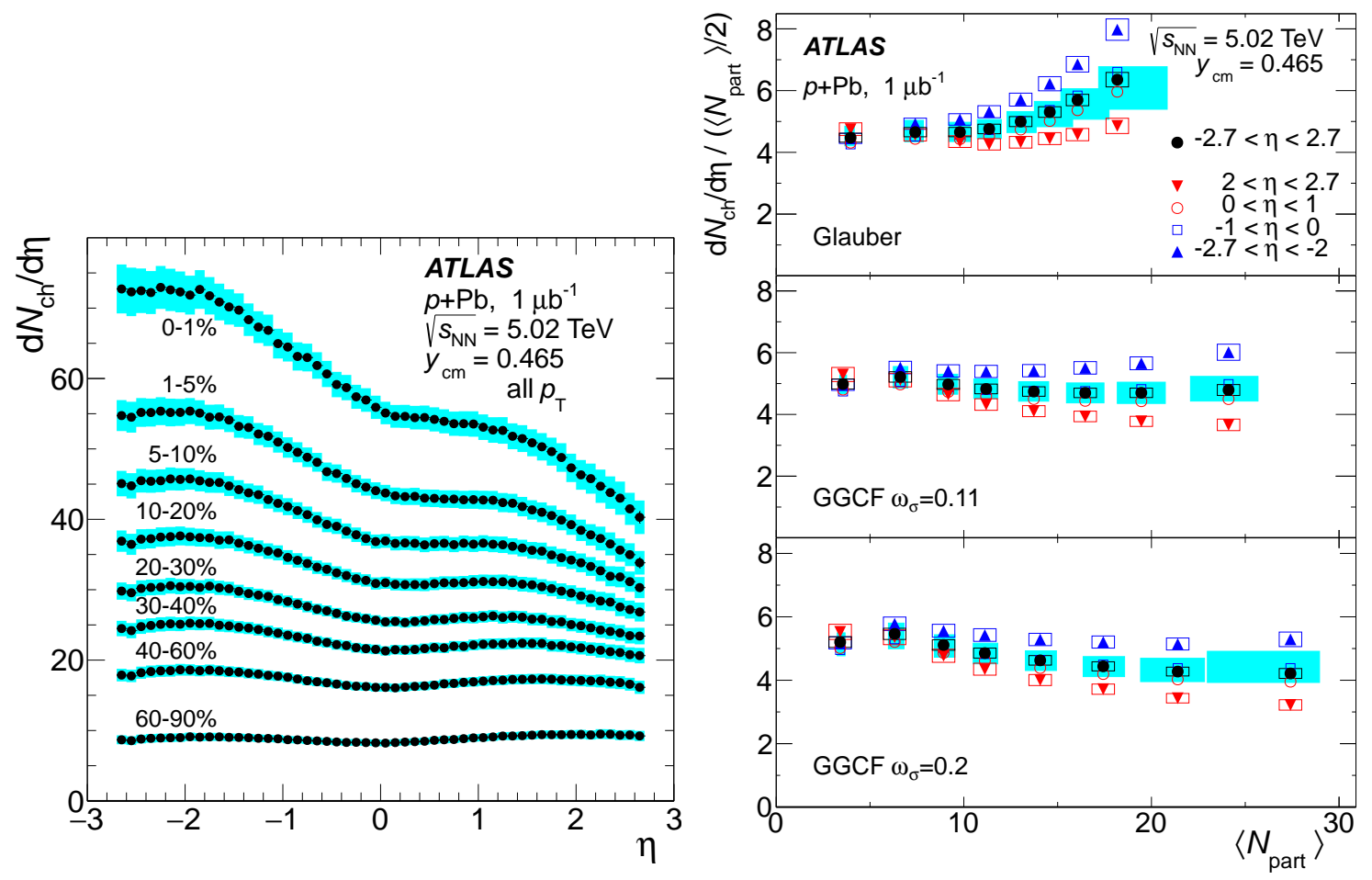

Fig. 2. Left: $\mathrm{d} N_{\mathrm{ch}} / \mathrm{d} \eta$ for charged particles with $p_{\mathrm{T}}>0 \mathrm{GeV}$. Statistical uncertainties, shown with vertical bars are typically smaller than the marker size. Shaded bands indicate systematic uncertainties on the measurements. Right: $\mathrm{d} N_{\mathrm{ch}} / \mathrm{d} \eta$ per participant pari for several $\eta$-regions for the three models of the geometry: the standard Glauber model (top panel), the GGCF model with $\omega_{\sigma}=0.11$ (middle panel) and GGCF with $\omega_{\sigma}=0.2$ (bottom panel). Taken from Ref. [30]

provide further insight.

\section{Initial stages of nuclear collisions}

ATLAS has performed a series of measurements sensitive to the initial geometry and particle production in $p+\mathrm{Pb}$ collisions. In these measurements the convention used is that negative $\eta$ corresponds to the direction of the $\mathrm{Pb}$ beam. The $\eta$ values are those in the laboratory frame, which differ froms from the centre-of-mass frame by a longitudinal boost factor of 0.465 in the direction of the proton. The centrality was determined by considering the total transverse energy in the ATLAS forward calorimeters in the direction of the $\mathrm{Pb}$ beam $(-4.9<\eta<-3.2), \sum E_{\mathrm{T}}^{\mathrm{Pb}}$. Centrality classes were defined by dividing the $\sum E_{\mathrm{T}}^{\mathrm{Pb}}$ distribution into successive partitions, each representing a fraction of the total distribution obtained in minimum-bias collisions [29, 30].

The most fundamental observable sensitive to the initial geometry is the total multiplicity of charged particles per unit pseudorapidity, $\mathrm{d} N_{\mathrm{ch}} / \mathrm{d} \eta$. The $\mathrm{d} N_{\mathrm{ch}} / \mathrm{d} \eta$ distributions were measured as a function of $\eta$ in bins of collision centrality. The left panel of Figure 2 shows the $\mathrm{d} N_{\mathrm{ch}} / \mathrm{d} \eta$ distributions as a function of $\eta$ for different centrality classes [30]. The multiplicity grows as the collision centrality is increased (smaller percentile value). This growth is larger in the $\mathrm{Pb}$ (backward) direction, indicating that the particle production receives a larger contribution from the number of struck participants in the nuclear target than from the number of times the projectile interacted. This is reminiscent of the Wounded Nucleon (WN) Model of particle production where the total multiplicity is proportional to the number of participant nucleons, $N_{\text {part }}[31]$. The validity of WN was tested more directly by scaling the $\mathrm{d} N_{\mathrm{ch}} / \mathrm{d} \eta$ values by the number of participant pairs, $\left\langle N_{\text {part }}\right\rangle / 2$. Three sets of $\left\langle N_{\text {part }}\right\rangle$ values were obtained: one set from a Glauber Model analysis and a two sets from a Glauber-Gribov Color Fluctuation (GGCF) Model [32] with different choices of the $\omega_{\sigma}$ 

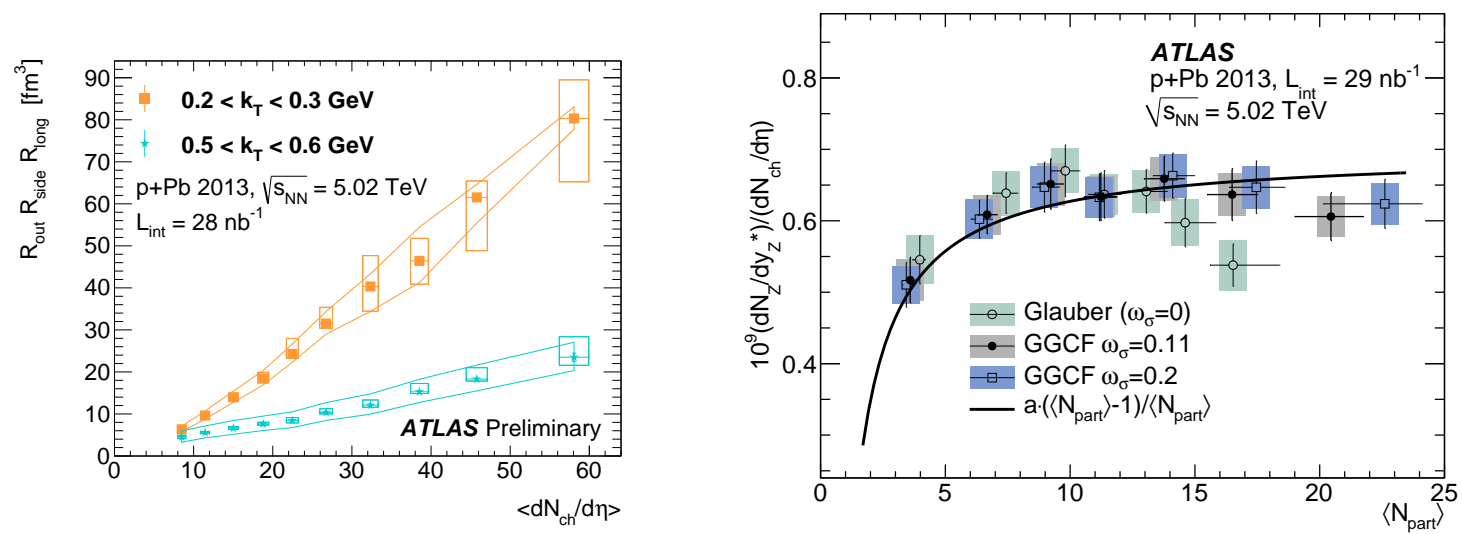

Fig. 3. Left: The product of the radii, $R_{\text {out }} R_{\text {side }} R_{\text {long }}$, for two $k_{\mathrm{T}}$ intervals plotted against the average multiplicity. The systematic uncertainties from the background description and pion identification are shown as error bands while the other systematic uncertainties are indicated by the height of the boxes. The horizontal error bars indicate the systematic uncertainty from $\left\langle\mathrm{d} N_{\mathrm{ch}} / \mathrm{d} \eta\right\rangle$. Taken from Ref. [38]. Right: The ratio of the $Z$ boson multiplicity to the inclusive charged particle multiplicity, $\left(\mathrm{d} N_{Z} / \mathrm{d} y_{Z}^{\star}\right) /\left\langle\mathrm{d} N_{\text {ch }} / \mathrm{d} \eta\right\rangle$, as a function of $\left\langle N_{\text {part }}\right\rangle$. A function of the form $a\left(\left\langle N_{\text {part }}\right\rangle-1\right) /\left\langle N_{\text {part }}\right\rangle$ is also shown. The normalization of $a$ is set based on the GGCF with $\omega_{\sigma}=0.11$ points. Statistical uncertainties are plotted as bars and systematic uncertainties as shaded boxes. Taken from Ref. [39].

parameter. Standard implementations of the Glauber Model [33] utilise a Monte Carlo procedure in which the probability for a pair of nucleons to interact is determined by whether they have geometric overlap, with their "sizes" fixed by the total inelastic nucleon-nucleon cross section. The GGCF is an extension of the traditional Glauber picture that accounts for the off-shell propagation of the nucleons between subsequent scatterings, and the implementation used here allows for per-event fluctuations in the nucleon-nucleon interaction probability [34-37], with the fluctuation spectrum assumed to follow a Normal distribution with a relative variance $\omega_{\sigma}=\left\langle\left(\sigma / \sigma_{\text {tot }}-1\right)^{2}\right\rangle$. This extension allows for configurations in which the projectile fluctuates to a large size resulting a longer tail to the $N_{\text {part }}$ distribution compared to the nominal Glauber, which has the effect of reducing the values of $\mathrm{d} N_{\mathrm{ch}} / \mathrm{d} \eta /\left(\left\langle N_{\text {part }}\right\rangle / 2\right)$ at large $\left\langle N_{\text {part }}\right\rangle$.

The right panel of Figure 2 shows the $\mathrm{d} N_{\mathrm{ch}} / \mathrm{d} \eta /\left(\left\langle N_{\text {part }}\right\rangle / 2\right)$ values for different $\eta$ ranges scaled by as a function of $\left\langle N_{\text {part }}\right\rangle$. The distributions indicate that the GGCF model with $\omega_{\sigma}=0.11$ is most consistent with the expectations of $\mathrm{WN}$, although this level of agreement depends significantly on the pseudorapidity region being considered, indicating that the models of initial geometry and particle production cannot be completely disentangled.

ATLAS has also reported a new measurement using Bose-Einstein correlations to provide interferometric three-dimensional imaging of the production source [38]. To reduce dependence on the geometric model, the $\left\langle\mathrm{d} N_{\mathrm{ch}} / \mathrm{d} \eta\right\rangle$ in a centrality class was used as a proxy for $\left\langle N_{\text {part }}\right\rangle$. The product of the extracted source radii is shown as a function of $\left\langle\mathrm{d} N_{\mathrm{ch}} / \mathrm{d} \eta\right\rangle$ in the left panel of Figure 3. The quantities are shown for two intervals in transverse momentum of the pair, $k_{\mathrm{T}}$, which are expected to be affected by the space-time evolution of the source differently. Both $k_{\mathrm{T}}$ ranges show the product of the three radii, expected to be proportional to the source volume, is linearly proportional to the $\left\langle\mathrm{d} N_{\mathrm{ch}} / \mathrm{d} \eta\right\rangle$.

The initial geometry is also expected to influence hard scattering rates, which are expected to scale with the average number of binary collisions $\left\langle N_{\text {coll }}\right\rangle$. In $p+\mathrm{Pb}$ collisions, $N_{\text {part }}=N_{\text {coll }}+1$. New ATLAS measurements of $Z$ [39] and $W$ boson production [40] (not shown) have confirmed this directly. The $Z$ boson yields scaled by $\left\langle\mathrm{d} N_{\mathrm{ch}} / \mathrm{d} \eta\right\rangle$ are shown in Figure 3. They exhibit the proper scaling including the behavior in more peripheral collisions where the (relative) difference between $N_{\text {coll }}$ and $N_{\text {part }}$ is more pronounced.

Measurements of inclusive jet production present a more complicated picture as the centrality dependence of the jet yield has been observed to correlate strongly with the hard scattering kinematics [41]. Specifically, the yield is observed to be highly suppressed in central collisions in a fashion that depends on the jet energy (not jet $p_{\mathrm{T}}$ and rapidity separately). This suggests a correlation between the $x$ of the parton in the proton and the effective size that determines its probability for inelastic scatterings with the nucleons in 

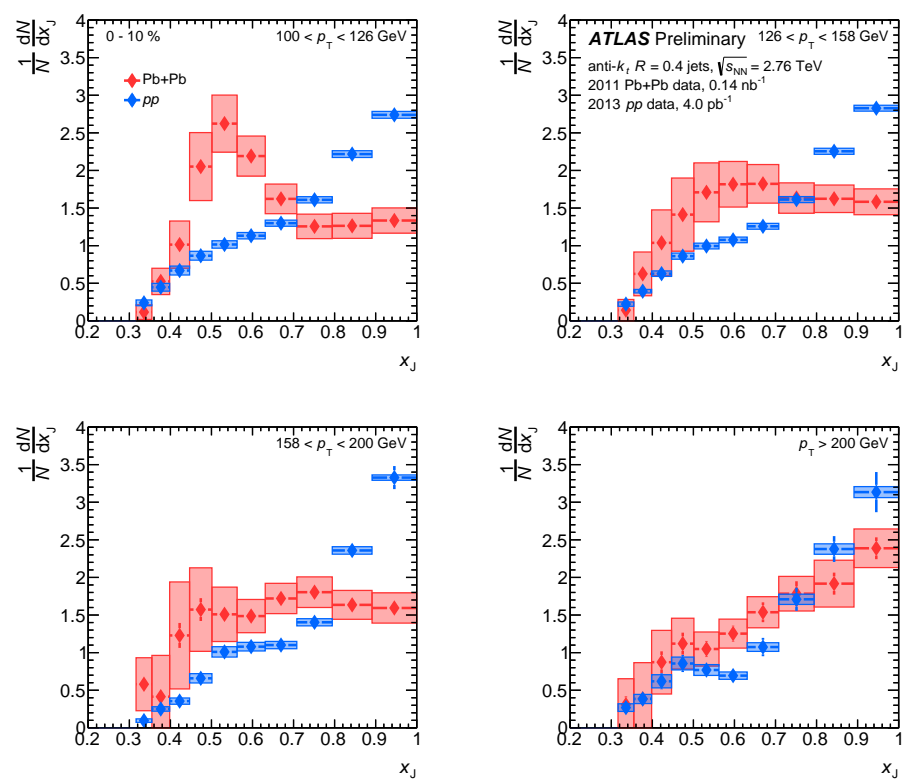

Fig. 4. The $\frac{1}{N} \frac{\mathrm{d} N}{\mathrm{~d} x_{\mathrm{J}}}$ distributions for different selections on $p_{\mathrm{T}_{1}}$, shown for the $0-10 \%$ centrality bin (red) and for $p p$ (blue). Statistical uncertainties are indicated by the error bars while systematic uncertainties are shown with shaded boxes. Taken from Ref. [44]

the nuclear target. A possible interpretation is that not only are the extensions present in GGCF relevant for the geometry, but that the color fluctuations may be correlated with the nucleon's partonic structure [42, 43].

\section{Hard probes of the quark-gluon plasma}

ATLAS has also performed new measurements sensitive to the physics of jet quenching. These include measurements of dijet $p_{\mathrm{T}}$ correlations in $\mathrm{Pb}+\mathrm{Pb}$ and $p p$ collisions at $\sqrt{s_{\mathrm{NN}}}=2.76 \mathrm{TeV}$ [44]. The momentum balance of the dijet system was expressed in terms of the variable $x_{\mathrm{J}} \equiv p_{\mathrm{T}_{2}} / p_{\mathrm{T}_{1}}$, where $p_{\mathrm{T}_{1}}$ and $p_{\mathrm{T}_{2}}$ are the transverse momenta of the jets with the highest and second highest $p_{\mathrm{T}}$ in the event, respectively. Measurements of $\frac{1}{N} \frac{\mathrm{d} N}{\mathrm{~d} x_{\mathrm{J}}}$ were performed as a function of $p_{\mathrm{T}_{1}}$ and collision centrality. Previous experimental results $[22,23,45]$ were not corrected to account for detector-specific experimental effects, in particular the resolution on the measured jet $p_{\mathrm{T}}$, which lead to distortions of the distributions. The ATLAS results reported here utilize a two-dimensional unfolding procedure to account for these effects. Thus they constitute the first measurement of dijet asymmetry that is free from such distortions and can be directly compared to theoretical calculations.

The results were obtained by measuring the two-dimensional $p_{\mathrm{T}_{1}}-p_{\mathrm{T}_{2}}$ distributions formed from pairs of jets with $p_{\mathrm{T}}>25 \mathrm{GeV},|y|<2.1$ and having $\Delta \phi>7 \pi / 8$. Despite this relatively tight requirement, the twodimensional distribution receives contributions from combinatoric pairs. These pairs arise from instances when another jet in the same event, but uncorrelated with the hard scattering, fulfills the requirements of the dijet pair selection through random association. Such jets may originate from independent hard scatterings but are mostly caused by upward fluctuations of the underlying event that are identified as jets. The measurement uses the observed contribution to the dijet $\Delta \phi$ distribution outside of the signal region to provide an estimate of the combinatoric contribution and subtracts this contribution bin-by-bin in $p_{\mathrm{T}_{1}}-p_{\mathrm{T}_{2}}$ prior to applying the unfolding procedure.

The $\frac{1}{N} \frac{\mathrm{d} N}{\mathrm{~d} x_{\mathrm{J}}}$ distributions obtained in central $\mathrm{Pb}+\mathrm{Pb}$ and $p p$ collisions are shown in Figure 4 for different intervals of $p_{\mathrm{T}_{1}}$. The distributions obtained in $p p$ collisions have the most probable value near $x_{\mathrm{J}} \sim 1$ indicating that dijet pairs that nearly balance in $p_{\mathrm{T}}$ are the most likely configuration. In contrast, events with $100<p_{\mathrm{T}_{1}}<126 \mathrm{GeV}$, the $\mathrm{Pb}+\mathrm{Pb}$ distributions develop a large peak at $x_{\mathrm{J}} \sim 0.5$, indicating that the most 


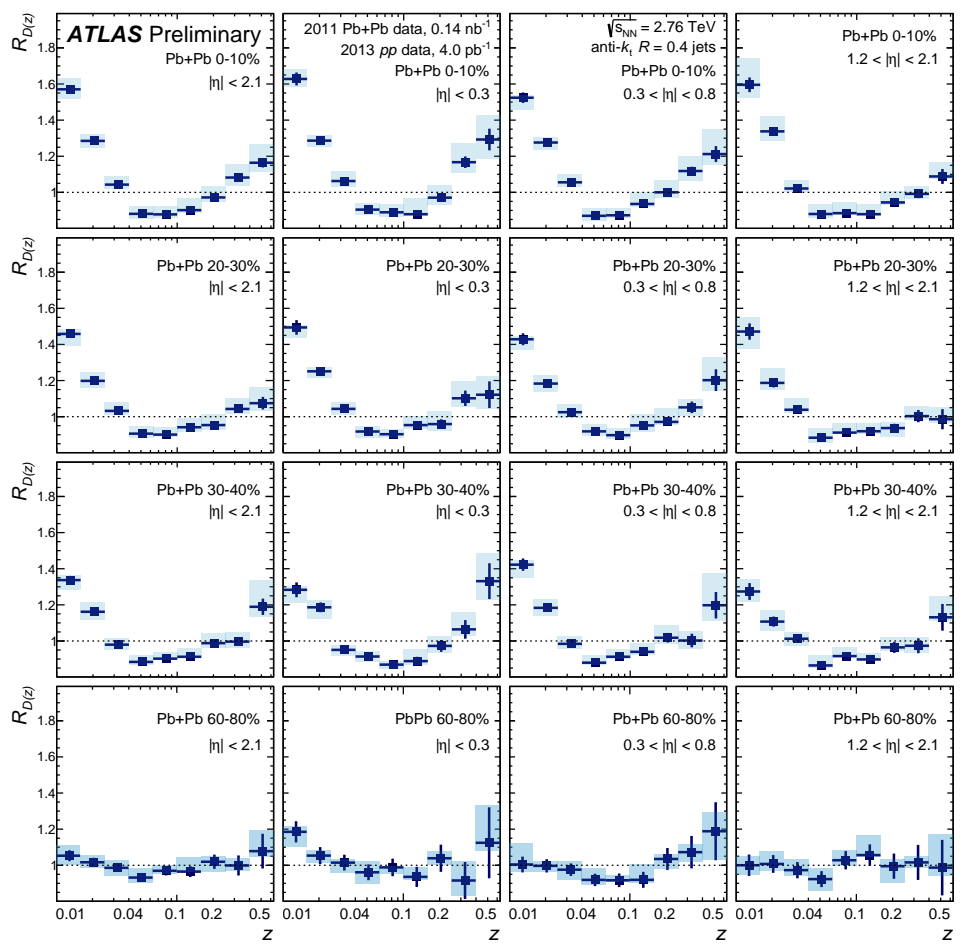

Fig. 5. The ratio of $\mathrm{D}(\mathrm{z})$ distributions measured in $\mathrm{Pb}+\mathrm{Pb}$ collisions to $\mathrm{D}(\mathrm{z})$ distributions measured in $p p$ collisions, $R_{D(z)}$. $R_{D(z)}$ distributions are evaluated in four different centrality bins (rows) and four different selections on jet pseudorapidity of jets with $100<$ $p_{\mathrm{T}}<398 \mathrm{GeV}$ (columns). The error bars on the data points indicate statistical uncertainties while the shaded bands indicate systematic uncertainties. Taken from Ref. [47].

probable configuration of dijet pairs in these events is where there is a large momentum imbalance. The modifications observed in the $\mathrm{Pb}+\mathrm{Pb}$ data gradually lessen with larger $p_{\mathrm{T}_{1}}$, although this evolution is much sharper than the $p_{\mathrm{T}}$ dependence of in single jet suppression, which is approximately logarithmic [46]. The modifications also gradually lessen in more peripheral centrality bins (not shown).

ATLAS has also performed new measurements of jet structure in $\mathrm{Pb}+\mathrm{Pb}$ and $p p$ collisions at $\sqrt{s_{\mathrm{NN}}}=$ $2.76 \mathrm{TeV}$ [47]. These results include observables related to the charged particle content within jets:

$$
D(z)=\frac{1}{N_{\text {jet }}} \frac{\mathrm{d} N_{\mathrm{ch}}}{\mathrm{d} z}, \quad D\left(p_{\mathrm{T}}^{\mathrm{ch}}\right)=\frac{1}{N_{\text {jet }}} \frac{\mathrm{d} N_{\mathrm{ch}}}{\mathrm{d} p_{\mathrm{T}}^{\mathrm{ch}}},
$$

where $p_{\mathrm{T}}^{\mathrm{ch}}$ refers the transverse momentum of a charged particle within $\Delta R=0.4$ of the jet and $z=$ $\left(p_{\mathrm{T}}^{\mathrm{ch}} / p_{\mathrm{T}}^{\text {jet }}\right) \cos \Delta R$. Previous measurements have shown that compared to references obtained from peripheral $\mathrm{Pb}+\mathrm{Pb}$ collisions or $p p$ collisions, the distributions in central $\mathrm{Pb}+\mathrm{Pb}$ collisions show an excess for $z<0.04$ and depletion for $0.04<z<0.2$. A possible enhancement at large $z$ was suggested by the data but was not significant beyond the uncertainties presented in each measurement, which were driven by statistical uncertainties in the reference. The new ATLAS measurement utilizes a high statistics $p p$ reference sample corresponding to $4.0 \mathrm{pb}^{-1}$ recorded in 2013. Compared to previous ATLAS results, this measurement also extends to lower $p_{\mathrm{T}}^{\text {ch }}$ (previously $2 \mathrm{GeV}$, now $1 \mathrm{GeV}$ ) and is now performed differentially in the $p_{\mathrm{T}}$ and pseudorapidity of the jet. Figure 5 shows the $D(z)$ distributions for different selections in jet pseudorapidity and centrality for jets with $p_{\mathrm{T}}>100 \mathrm{GeV}$. In the most central collisions, an enhancement of up to $60 \%$ in the particle yield at the lowest $z$ values are observed and the enhancement at large $z$ is observed to be significant. The modifications at low $z$ do not change appreciably with jet $\eta$. However the modifications at high $z$ decrease from greater that $20 \%$ for jets with $|\eta|<0.3$ to approximately $7 \%$ for jets with $1.2<|\eta|<2.1$. The 
variation of $D(z)$ with $p_{\mathrm{T}}^{\text {jet }}$ (not shown) exhibits a similar trend. The $D\left(p_{\mathrm{T}}^{\text {ch }}\right)$ exhibits nearly identical trends in all cases to the $D(z)$ distributions.

The dependence of the $D(z) / D\left(p_{\mathrm{T}}^{\mathrm{ch}}\right)$ modifications on the jet kinematics suggest that the populations of jets identified by these selections possess different parton showers and are differently affected by the medium. This is consistent with the supposition that jets at larger $\eta$ receive a larger contribution from parton showers originating from quarks as opposed to gluons and that such quark jets may lose less energy in the medium [48]. Information about the potential dependence of energy loss on parton flavor is also present in recent ATLAS measurements of the rapidity dependence of nuclear modification factor, $R_{\mathrm{AA}}$ for jets [46] and single hadrons [49]. However, the competing effects - such as the steeper spectral slope larger $\eta$ - reduce the sensitivity of these observables to the quark/gluon distinction. In principle the $p_{\mathrm{T}_{1}}$ dependence of the dijet $\frac{1}{N} \frac{\mathrm{d} N}{\mathrm{~d} x_{\mathrm{J}}}$ distributions discussed above may also be sensitive to such effects through how the pair composition ( e.g. quark-quark, quark-gluon etc.) evolves with $p_{\mathrm{T}_{1}}$. New theoretical calculations have been successful in describing the rapidity dependence of the jet $R_{\mathrm{AA}}$ [50]. The dijet and jet structure measurements discussed here present a new series of benchmarks to test specific details of the energy loss implemented in such models.

\section{Conclusion}

These proceedings have presented a set of recent measurements performed by ATLAS that address some of the most fundamental questions in the field of ultra-relativistic heavy-ion collisions. Measurements of two-particle correlations in $p p$ collisions at $\sqrt{s}=2.76$ and $13 \mathrm{TeV}$ were presented. The results show a second harmonic modulation to the particle yields that is typically associated with hydrodynamic expansion in ionion collisions. This modulation exhibits almost no dependence on collision energy or total event activity, indicating that phenomenon giving rise to the correlation is an ever-present feature of the strong interaction at the $\mathrm{TeV}$ scale. Possible explanations of the phenomenon, including hydrodynamics, require a detailed description of the initial collision geometry in these systems. ATLAS has presented measurements from $p+\mathrm{Pb}$ collisions at $\sqrt{s_{\mathrm{NN}}}=5.02 \mathrm{TeV}$, which provide direct information on the geometry and its relationship with particle production. Measurements of the centrality dependence of the total charged particle multiplicity suggest that extensions to the canonical Glauber Model - Glauber-Gribov Color Fluctuations - may be necessary to obtain a detailed description of the geometry. The fluctuations introduced by this extension are likely to affect the distribution of spacial eccentricities, which have traditionally been the initial conditions to hydrodynamical models of the system's evolution. Finally, new results on jet quenching were presented. Measurements of dijet momentum balance and jet structure in $\mathrm{Pb}+\mathrm{Pb}$ collisions at $\sqrt{s_{\mathrm{NN}}}=2.76 \mathrm{TeV}$ provide new benchmarks for theoretical models in this rapidly developing topic. The dependence of these observables on the jet $p_{\mathrm{T}}$ and rapidity suggest that, for the first time, a dependence of the energy loss on parton flavor (quark vs gluon) may be directly observable.

\section{References}

[1] I. Arsene, et al., (BRAHMS Collaboration), Nucl. Phys. A757 (2005) 1-27. arXiv:nucl-ex/0410020.

[2] B. Back, et al., (PHOBOS Collaboration), Nucl. Phys. A757 (2005) 28-101. arXiv:nucl-ex/0410022.

[3] J. Adams, et al., (STAR Collaboration), Nucl. Phys. A 757 (2005) 102-183. arXiv:nucl-ex/0501009.

[4] K. Adcox, et al., (PHENIX Collaboration), Nucl. Phys. A 757 (2005) 184-283. arXiv:nucl-ex/0410003.

[5] ATLAS Collaboration, Phys. Rev. Lett. 110 (18) (2013) 182302. arXiv:1212.5198 [hep-ex].

[6] ATLAS Collaboration, Phys. Rev. C90 (4) (2014) 044906. arXiv:1409.1792 [hep-ex].

[7] ATLAS Collaboration, Phys. Lett. B725 (2013) 60-78. arXiv:1303.2084 [hep-ex].

[8] ALICE Collaboration, Phys. Lett. B719 (2013) 29-41. arXiv:1212.2001 [nucl-ex].

[9] ALICE Collaboration, Phys. Lett. B726 (2013) 164-177. arXiv:1307.3237 [nucl-ex].

[10] ALICE Collaboration, Phys. Rev. C90 (5) (2014) 054901. arXiv:1406.2474 [nucl-ex].

[11] ALICE Collaboration, Phys. Lett. B753 (2016) 126-139. arXiv:1506.08032 [nucl-ex].

[12] CMS Collaboration, Phys. Lett. B724 (2013) 213-240. arXiv:1305.0609 [nucl-ex].

[13] CMS Collaboration, Phys. Lett. B742 (2015) 200-224. arXiv:1409.3392 [nucl-ex].

[14] CMS Collaboration, Phys. Rev. Lett. 115 (1) (2015) 012301. arXiv:1502.05382 [nucl-ex].

[15] A. Adare, et al., (PHENIX Collaboration), Phys. Rev. Lett. 111 (21) (2013) 212301. arXiv:1303.1794 [nucl-ex].

[16] S. S. Adler, et al., (PHENIX Collaboration), Phys. Rev. Lett. 91 (2003) 072301. arXiv:nucl-ex/0304022. 
[17] J. Adams, et al., (STAR Collaboration), Phys. Rev. Lett. 91 (2003) 172302. arXiv:nucl-ex/0305015.

[18] I. Arsene, et al., (BRAHMS Collaboration), Phys. Rev. Lett. 91 (2003) 072305. arXiv:nucl-ex/0307003.

[19] B. B. Back, et al., (PHOBOS Collaboration), Phys. Rev. Lett. 94 (2005) 082304. arXiv:nucl-ex/0405003.

[20] C. Adler, et al., (STAR Collaboration), Phys. Rev. Lett. 90 (2003) 082302. arXiv:nucl-ex/0210033.

[21] A. Adare, et al., (PHENIX Collaboration), Phys. Rev. C78 (2008) 014901. arXiv:0801.4545 [nucl-ex].

[22] ATLAS Collaboration, Phys. Rev. Lett. 105 (2010) 252303. arXiv:1011.6182 [hep-ex].

[23] CMS Collaboration, Phys. Rev. C84 (2011) 024906. arXiv:1102.1957 [nucl-ex].

[24] ATLAS Collaboration, JINST 3 (2008) S08003.

[25] ATLAS Collaboration, to appear in Phys. Rev. Lett. arXiv:1509.04776 [hep-ex].

[26] B. Alver, G. Roland, Phys. Rev. C81 (2010) 054905, [Erratum: Phys. Rev.C82,039903(2010)]. arXiv:1003.0194 [nucl-th].

[27] N. N. Ajitanand, J. M. Alexander, P. Chung, P. Danielewicz, W. G. Holzmann, M. Issah, R. A. Lacey, A. Shevel, A. Taranenko, Phys. Rev. C 72 (2005) 011902.

[28] P. Bozek, Phys. Rev. C85 (2012) 014911. arXiv:1112.0915 [hep-ph].

[29] ATLAS Collaboration, Phys. Lett. B 707 (2012) 330-348. arXiv:1108.6018 [hep-ex].

[30] ATLAS Collaboration, to appear in Phys. Lett. B. arXiv:1508.00848 [hep-ex].

[31] A. Bialas, M. Bleszynski, W. Czyz, Nucl. Phys. B111 (1976) 461.

[32] V. N. Gribov, Sov. Phys. JETP 29 (1969) 483-487.

[33] M. L. Miller, K. Reygers, S. J. Sanders, P. Steinberg, Ann. Rev. Nucl. Part. Sci. 57 (2007) 205-243. arXiv:nucl-ex/0701025 [nucl-ex].

[34] G. Baym, B. Blättel, L. L. Frankfurt, H. Heiselberg, M. Strikman, Phys. Rev. Lett. 67 (1991) 2946-2949.

[35] G. Baym, B. Blättel, L. L. Frankfurt, H. Heiselberg, M. Strikman, Phys. Rev. D 47 (1993) 2761-2772.

[36] V. Guzey, M. Strikman, Phys. Lett. B633 (2006) 245-252, [Phys. Lett.B663,456(2008)]. arXiv:hep-ph/0505088 [hep-ph].

[37] M. Alvioli, M. Strikman, Phys. Lett. B722 (2013) 347-354. arXiv:1301.0728 [hep-ph].

[38] ATLAS Collaboration, ATLAS-CONF-2015-054 (2015). http://cds.cern.ch/record/2055675

[39] ATLAS Collaboration, Phys. Rev. C92 (4) (2015) 044915. arXiv:1507.06232 [hep-ex].

[40] ATLAS Collaboration, ATLAS-CONF-2015-056 (2015). http://cds . cern.ch/record/2055677

[41] ATLAS Collaboration, Phys. Lett. B748 (2015) 392-413. arXiv:1412.4092 [hep-ex].

[42] M. Alvioli, L. Frankfurt, V. Guzey, M. Strikman, Phys. Rev. C90 (2014) 034914. arXiv:1402.2868 [hep-ph].

[43] M. Alvioli, B. A. Cole, L. Frankfurt, D. V. Perepelitsa, M. Strikman, Phys. Rev. C93 (1) (2016) 011902. arXiv:1409.7381 [hep-ph].

[44] ATLAS Collaboration, ATLAS-CONF-2015-052 (2015). http://cds.cern.ch/record/2055673

[45] CMS Collaboration, Phys. Lett. B712 (2012) 176-197. arXiv:1202.5022 [nucl-ex].

[46] ATLAS Collaboration, Phys. Rev. Lett. 114 (7) (2015) 072302. arXiv:1411.2357 [hep-ex].

[47] ATLAS Collaboration, ATLAS-CONF-2015-055 (2015). http://cds . cern. ch/record/2055676

[48] M. Spousta, B. Cole. arXiv:1504.05169 [hep-ph].

[49] ATLAS Collaboration, JHEP 09 (2015) 050. arXiv:1504.04337 [hep-ex].

[50] Y.-T. Chien, I. Vitev. arXiv:1509.07257 [hep-ph]. 